\section{The Optimal Projection Equations for Fixed-Order Sampled-Data Dynamic Compensation with Computation Delay}

\author{
DENNIS S. BERNSTEIN, LAWRENCE D. DAVIS, AND \\ SCOTT W. GREELEY
}

\begin{abstract}
For an LQG-type sampled-data regulator problem which accounts for computational delay and utilizes an averaging $\mathbf{A} / \mathbf{D}$ device, the equivalent discrete-time problem is shown to be of increased order due to the inclusion of delayed measurement states. The optimal projection equations for reduced-order, discrete-time compensation are applied to the augmented problem to characterize low-order controllers. The design resulis are illustrated on a tenth-order flexible beam example.
\end{abstract}

\section{INTRODUCTION}

Classical sampled-data control theory has been extensively developed [1]-[7] and is widely used in practical applications. Sampled-data design based upon modern optimal control theory has also been developed, although to a considerably lesser extent [8]-[14]. The goals of the present note are twofold. First, for an LQG-type sampled-data regulator problem which explicitly accounts for computational delay, we obtain an equivalent discrete-time problem (Theorem 2.1 and Corollary 2.1). The timing diagram in Fig. 1 illustrates the unavoidable delay in the feedback loop (see Section II for notation). ${ }^{1}$ A salient feature of this problem is that rather than replace the continuous-time white noise measurement model by a discrete-time version (which is often done in the literature since continuous-time white noise cannot be sampled), we employ an averaging-type A/D device as in [8, p. 82] [see (2.5)].

The second goal of the note is to present a novel design procedure which is applicable to the equivalent discrete-time problem, and which thus directly accounts for the delay effects. Since the discrete-time model is of augmented order $n+l$ ( $n=$ number of plant states, $l=$ number of measurements), it seems natural to seek dynamic feedback of reduced order. To this end, we apply the optimal projection equations for discretetime dynamic compensation to the equivalent discrete-time problem to characterize optimal controllers of order $n_{c} \leq n+l$. These equations, which were previously derived in [17] for the continuous-time case, are discussed in [15], [16]. Note that, in practice, the computational delay (and, hence, sample interval) in real-time controller implementation depends directly upon the controller order $n_{c}$. For example, by reducing $n_{c}$ the sample rate can effectively be increased. Thus, the engineering tradeoffs of performance versus controller order and sample interval can be investigated using the results of this note.

This note also includes formulas for integrals of matrix exponentials arising in the sampled-data/discrete-time conversion, along with an algorithm for solving the optimal projection equations. The results are applied to a tenth-order flexible beam example.

\section{Sampled-Data Problem and Equivalent Discrete-Time FORMULATION}

The following notation and definitions will be used throughout.

$$
\begin{array}{ll}
I_{r}, 0_{r \times s}, 0_{r} & r \times r \text { identity matrix, } r \times s \text { zero matrix, } 0_{r \times r} \\
Z_{(i, j)} & (i, j) \text { element of matrix } Z
\end{array}
$$

Manuscript received October 4, 1985; revised May 12, 1986. This work was supported in part by the Air Force Office of Scientific Research under Contracts AFOSR F49620$84-\mathrm{C}-0015, \mathrm{~F} 49620-86-\mathrm{C}-0002$, and F49620-86-C-0038.

The authors are with the Controls Analysis and Synthesis Group, Government Aerospace Systems Division, Harris Corporation, Melboume, FL 32902

IEEE Log Number 8609872.

' For simplicity, the timing diagram Fig. 1 applies to the case in which the compensator is strictly proper. In the note the results are stated for the more general case in which a direct (static) feedthrough term $D_{c} \hat{y}(k)$ is included.
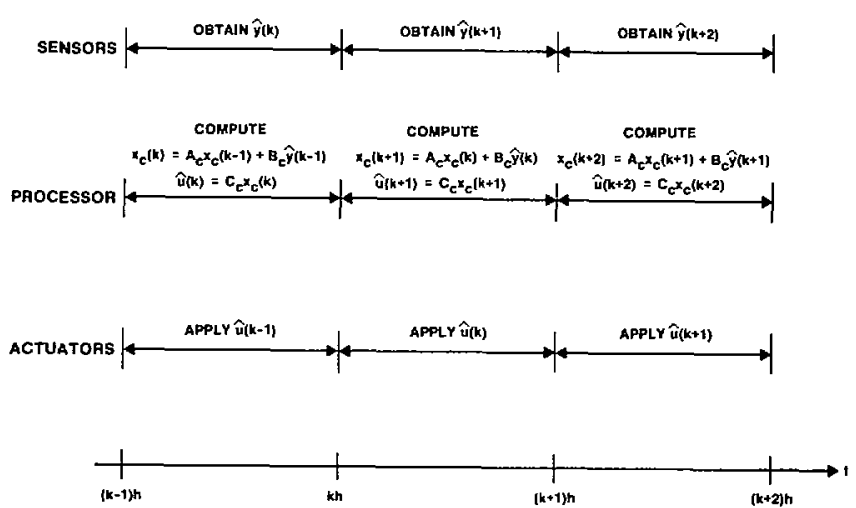

Fig. 1. Timing diagram for sampled-data controller.

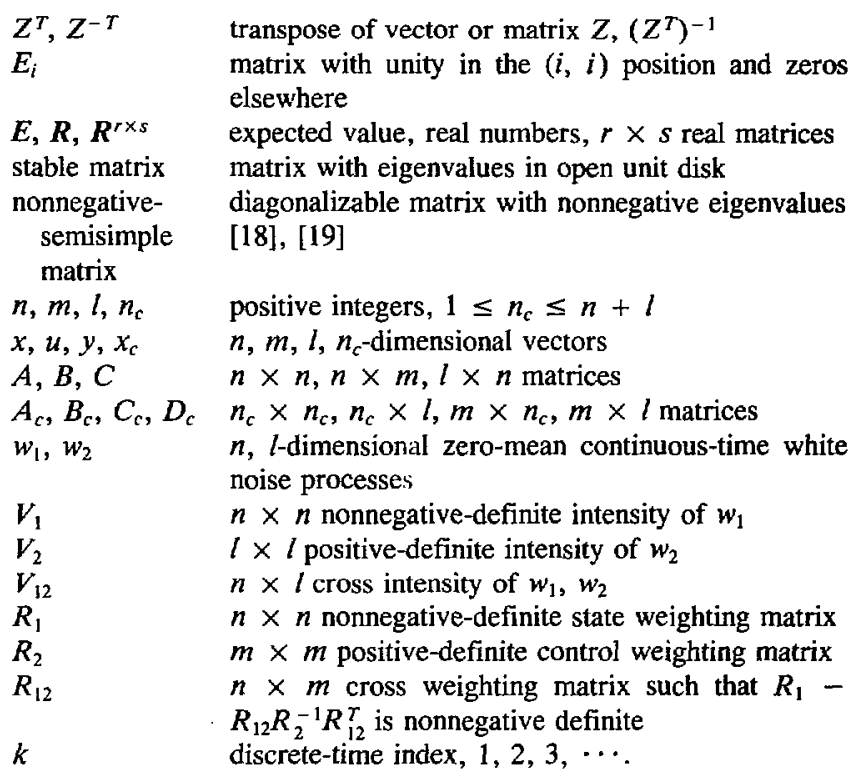

In the statement of the sampled-data control problem the sample interval $h$ and the controller order $n_{\mathrm{c}}$ are fixed and the optimization is performed over the controller parameters $\left(A_{c}, B_{c}, C_{c}, D_{c}\right)$. For design tradeoff studies $h$ and $n_{c}$ can be varied and the problem can be solved for each pair of values of interest.

\section{Fixed-Order, Sampled-Data Dynamic-Compensation Problem}

Given the $n$ th-order continuous-time system

$$
\dot{x}(t)=A x(t)+B u(t)+w_{1}(t)
$$

with continuous-time measurements

$$
y(t)=C x(t)+D u(t)+w_{2}(t)
$$

design an $n_{c}$ th-order discrete-time compensator

$$
\begin{gathered}
x_{c}(k+1)=A_{c} x_{c}(k)+B_{c} \hat{y}(k), \\
\hat{u}(k)=C_{c} x_{c}(k)+D_{c} \hat{y}(k),
\end{gathered}
$$

which, with $\mathrm{A} / \mathrm{D}$ averaged measurements

$$
\hat{y}(k) \triangleq \frac{1}{h} \int_{(k-1) h}^{k h} y(t) d t
$$


and D/A zero-order-hold controls

$$
u(t)=\hat{u}(k), \quad t \in[k h,(k+1) h),
$$

minimizes the performance criterion

$$
\begin{aligned}
J\left(A_{c}, B_{c}, C_{c}, D_{c}\right) \triangleq \lim _{t \rightarrow \infty} E \frac{1}{t} \\
\cdot \int_{0}^{l}\left[x(s)^{T} R_{1} x(s)+2 x(s)^{T} R_{12} u(s)+u(s)^{T} R_{2} u(s)\right] d s .
\end{aligned}
$$

The main result of this section concerns propagation of the plant and digitized measurements over one time step. For notational convenience, define

$$
H(s) \triangleq \int_{0}^{s} e^{A r} d r
$$

Theorem 2.1: For the fixed-order, sampled-data dynamic-compensation problem, the plant dynamics (2.1), averaged measurements (2.5) and performance criterion (2.7) have the equivalent discrete-time representations

$$
\begin{gathered}
x^{\prime}(k+1)=A^{\prime} x^{\prime}(k)+B^{\prime} \hat{u}(k)+w_{1}^{\prime}(k), \\
\hat{y}(k)=C^{\prime} x^{\prime}(k-1)+D^{\prime} \hat{u}(k-1)+w_{2}^{\prime}(k-1),
\end{gathered}
$$

$J\left(A_{c}, B_{c}, C_{c}, D_{c}\right)=\delta+\lim _{k \rightarrow \infty} E\left[x^{\prime}(k)^{T} R_{1}^{\prime} x^{\prime}(k)\right.$

$$
\left.+2 x^{\prime}(k)^{T} R_{12}^{\prime} \hat{u}(k)+\hat{u}(k)^{T} R_{2}^{\prime} \hat{a}(k)\right]
$$

where

$$
x^{\prime}(k) \triangleq x(k h), \delta \triangleq \frac{1}{h} \mathrm{tr} \int_{0}^{h} \int_{0}^{s} e^{A r} V_{1} e^{A} T_{r} R_{1} d r d s,
$$

$A^{\prime} \triangleq e^{A h}, B^{\prime} \triangleq H(h) B, C^{\prime} \triangleq \frac{1}{h} C H(h), D^{\prime} \triangleq \frac{1}{h} C \int_{0}^{h} H(s) d s B+D ;$

$w_{1}^{\prime}(k)$ and $w_{2}^{\prime}(k)$ are zero-mean, white noise processes with

$$
E\left\{\left[\begin{array}{l}
w_{1}^{\prime}(k) \\
w_{2}^{\prime}(k)
\end{array}\right]\left[\begin{array}{l}
w_{1}^{\prime}(k) \\
w_{2}^{\prime}(k)
\end{array}\right]\right\}=\left[\begin{array}{cc}
V_{1}^{\prime} & V_{12}^{\prime} \\
V_{12}^{\prime} T & V_{2}^{\prime}
\end{array}\right],
$$

where

$$
\begin{aligned}
& V_{i} \triangleq \int_{0}^{h} e^{A s} V_{1} e^{A_{s}} d s, V_{12}^{\prime} \triangleq \frac{1}{h} \int_{0}^{h} e^{A s} V_{1} H^{T}(s) d s C^{T}+\frac{1}{h} H(h) V_{12}, \\
& V_{2}^{\prime} \triangleq \frac{1}{h} V_{2}+\frac{1}{h^{2}} C \int_{0}^{h} H(s) V_{1} H^{T}(s) d s C^{T} \\
& \quad+\frac{1}{h^{2}} C \int_{0}^{h} H(s) d s V_{12}+\frac{1}{h^{2}} V_{12}^{T} \int_{0}^{h} H^{T}(s) d s C^{T} ;
\end{aligned}
$$

and

$$
R_{i}^{\prime} \triangleq \frac{1}{h} \int_{0}^{h} e^{A T_{s}} R_{1} e^{A s} d s, R_{12} \triangleq \frac{1}{h} \int_{0}^{h} e^{A} T_{s} R_{1} H(s) d s B+\frac{1}{h} H^{T}(h) R_{12},
$$$$
R_{2}^{\prime} \triangleq R_{2}+\frac{1}{h} B^{\tau} \int_{0}^{h} H^{T}(s) R_{1} H(s) d s B
$$

$$
+\frac{1}{h} B^{T} \int_{0}^{h} H^{T}(s) d s R_{12}+\frac{1}{h} R_{12}^{T} \int_{0}^{h} H(s) d s B
$$

The proof of this theorem is a straightforward calculation involving standard techniques, and hence is omitted. The result is more comprehensive than previous work, however, and includes several results as special cases. For example, the expressions for $A^{\prime}$ and $B^{\prime}$ are standard; $C^{\prime}$ is given by (10.9), [8, p. 83]; $R_{1}^{\prime}, R_{12}^{\prime}$, and $R_{2}^{\prime}$ are given in [10], [12]; and $V_{1}^{\prime}, V_{12}^{\prime}$, and $V_{2}^{\prime}$ can be found in $\left[8\right.$, p. 85]. The expressions for $\delta$ and $D^{\prime}$ appear to be new.

Note that the averaged measurements depend upon delayed samples of the state. By augmenting the discretized state equation (2.8) to include these measurements, it is possible to state the original sampled-data problem as a discrete-time problem.

Corollary 2.1: With the notation

$$
\begin{aligned}
& \hat{x}(k) \triangleq\left[\begin{array}{c}
x^{\prime}(k) \\
\hat{y}(k)
\end{array}\right], \hat{A} \triangleq\left[\begin{array}{cc}
A^{\prime} & 0_{n \times t} \\
C^{\prime} & 0_{l}
\end{array}\right], \hat{B} \triangleq\left[\begin{array}{c}
B^{\prime} \\
D^{\prime}
\end{array}\right], \\
& \hat{C} \triangleq\left[\begin{array}{ll}
0_{l \times n} & I
\end{array}\right], \hat{w}(k) \triangleq\left[\begin{array}{l}
w_{1}^{\prime}(k) \\
w_{2}^{\prime}(k)
\end{array}\right], \hat{V} \triangleq\left[\begin{array}{cc}
V_{1}^{\prime} & V_{12}^{\prime} \\
V_{12}^{\prime T} & V_{2}^{\prime}
\end{array}\right], \\
& \hat{R}_{1} \triangleq\left[\begin{array}{cc}
R_{i}^{\prime} & 0_{n \times 1} \\
0_{l \times n} & 0_{l}
\end{array}\right], \hat{R}_{12} \triangleq\left[\begin{array}{c}
R_{j 2}^{\prime} \\
0_{l \times m}
\end{array}\right], \hat{R}_{2} \triangleq R_{2}^{\prime}
\end{aligned}
$$

the fixed-order, sampled-data dynamic-compensation problem is equivalent to the following discrete-time problem. Given the $(n+l)$ th-order discrete-time system

$$
\hat{x}(k+1)=\hat{A} \hat{x}(k)+\hat{B} \hat{u}(k)+\hat{w}(k)
$$

with discrete-time measurements

$$
\hat{y}(k)=\hat{C} \hat{x}(k)
$$

design an $n_{c}$ th-order discrete-time compensator of the form (2.3), (2.4), which minimizes

$$
\begin{aligned}
J\left(A_{c}, B_{c}, C_{c}, D_{c}\right)=\delta+\lim _{k \rightarrow \infty} E & {\left[\hat{x}(k)^{T} \hat{R}_{1} \hat{x}(k)\right.} \\
& \left.+2 \hat{x}(k)^{r} \hat{R}_{12} \hat{u}(k)+\hat{u}(k)^{\top} \hat{R}_{2} \hat{u}(k)\right] .
\end{aligned}
$$

Remark 2.l: The equivalent cost (2.13) involves a constant offset $\delta$ which serves as a lower bound on the sampled-data performance, i.e., a "discretization floor." Note that

$$
\delta=\frac{h}{2} \operatorname{tr} V_{1} R_{1}+O\left(h^{2}\right)
$$

Remark 2.2: Although the measurements $\hat{y}(k)$ are noise free, the singularity is not so serious as singular measurement noise in the continuous-time case where the Kalman filter gains are expressed in terms of the inverse of the measurement noise intensity. In the discrete-time case, rather, it is required that $\bar{V}+\overline{C Q C}^{T}$ be invertible, where $\bar{V}$ is the measurement noise covariance (see $[11$, p. 530], or [15], [16]).

Remark 2.3: The increase in plant order from $n$ to $n+l$ is due to the computational delay and A/D process. Since discrete-time LQG theory yields a possibly unwieldy $(n+i)$ th-order controller, we seek "reducedorder" controllers. Note that in this context an $n$ th-order controller can be regarded as being of reduced order.

Remark 2.4: As pointed out in [10], particular choices of the sample interval $h$ may result in a loss of controllability and observability for the equivalent discrete-time problem. Hence, these properties must be verified before applying control design methods.

\section{Application of the Optimal Projection EQuations to the EQUIVALENT DISCRETE-TIME PROBLEM}

We now apply the optimal projection equations for discrete-time dynamic compensation to the equivalent discrete-time problem. The following easily proved lemma will be needed.

Lemma 3.1: Let $r \in \boldsymbol{R}^{(n+l) \times(n+l)}$. Then

$$
\begin{gathered}
\tau^{2}=\tau, \\
\rho(\tau)=n_{c}
\end{gathered}
$$


if and only if there exist $G, \Gamma \in R^{n_{c} \times(n+l)}$ such that

$$
\begin{gathered}
G^{T} \Gamma=\tau, \\
\Gamma G^{T}=I_{n_{c}} .
\end{gathered}
$$

Furthermore, $G$ and $\Gamma$ are unique to a change of basis in $\boldsymbol{R}^{\boldsymbol{n}_{\mathrm{c}} \text {. }}$

Call $G$ and $\Gamma$ satisfying (3.3), (3.4) a projective factorization of $\tau$. Furthermore, for $n \times n$ nonnegative-definite matrices $Q$ and $\mathcal{P}$, define the set of contragrediently diagonalizing transformations

$$
\mathcal{C}(\mathcal{Q}, \mathcal{P}) \triangleq\left\{\Psi \in R^{n \times n}: \Psi^{-1} \mathcal{Q} \Psi^{-T} \text { and } \Psi^{T} \mathcal{Q} \Psi \text { are diagonal }\right\} .
$$

It follows from [19, Theorem $6.2 .5, \mathrm{p}, 123]$ that $\mathcal{C}(Q, P)$ is always nonempty. This set does not, however, have a unique element since basis rearrangements and sign transpositions may be incorporated into $\Psi$. Further nonuniqueness arises if $2 \mathbb{O}$ has repeated eigenvalues.

To guarantee that $J$ is finite and independent of initial conditions, we restrict our consideration to the (open) set

$S \triangleq\left\{\left(A_{\varepsilon}, B_{c}, C_{c}, D_{c}\right):\left[\begin{array}{cc}\hat{A}+\hat{B} D_{c} \hat{C} & \hat{B} C_{c} \\ B_{c} \hat{C} & A_{c}\end{array}\right]\right.$

is stable and $\left(A_{c}, B_{c}, C_{c}\right)$ is minimal $\}$.

For the design problem it is required that $S$ be nonempty, i.e., that the augmented system be stabilizable. We also require the notation

$$
\begin{gathered}
\tilde{R}_{2} \triangleq \hat{R}_{2}+\hat{B}^{T} P \hat{B}, \widetilde{V}_{2} \triangleq \hat{C} Q \hat{C}^{T}, \tau_{\perp} \triangleq I_{n+1}-\tau, \\
A_{Q} \triangleq \hat{A}-\hat{A} Q \hat{C}^{T} \tilde{V}_{2}^{-1} \hat{C}, A_{P} \triangleq \hat{A}-\hat{B} \tilde{R}_{2}^{-1}\left(\hat{B}^{T} P \hat{A}+\hat{R}_{12}^{T}\right), \\
\Sigma_{Q} \triangleq\left(\hat{A} Q \hat{C}^{T}+\hat{B} D_{c} \widetilde{V}_{2}\right) \tilde{V}_{2}^{-1}\left(\hat{A} Q \hat{C}^{T}+\hat{B} D_{c} \tilde{V}_{2}\right)^{T}, \\
\Sigma_{P} \triangleq\left(\hat{B}^{T} P \hat{A}+\hat{R}_{12}^{T}+\tilde{R}_{2} D_{c} \hat{C}\right)^{r} \tilde{R}_{2}^{-1}\left(\hat{B}^{T} P \hat{A}+\hat{R}_{12}^{T}+\tilde{R}_{2} D_{c} \hat{C}\right),
\end{gathered}
$$

$M \triangleq\left[\begin{array}{c}I_{n+1} \\ D_{c} \hat{C}\end{array}\right], \hat{M} \triangleq\left[\begin{array}{c}I_{n+1} \\ -\tilde{R}_{2}^{-1}\left(\hat{B}^{T} P \hat{A}+\hat{R}_{12}^{T}\right)\end{array}\right]$,

$\tilde{R} \triangleq\left[\begin{array}{cc}\hat{R}_{1} & \hat{R}_{12} \\ \hat{R}_{12}^{T} & \hat{R}_{2}\end{array}\right]$.

Theorem 3.1: Suppose $\left(A_{\mathfrak{c}}, B_{c}, C_{c}, D_{c}\right) \in S$ solves the fixed-order, sampled-data dynamic-compensation problem. Then there exist $(n+l)$ $\times(n+l)$ nonnegative-definite matrices $Q, P, \hat{Q}$, and $\hat{P}$ such that $\boldsymbol{A}_{\boldsymbol{c}}$, $B_{c}, C_{c}$, and $D_{c}$ are given by

$$
\begin{aligned}
& A_{c}=\Gamma\left[\hat{A}-\hat{A} Q \hat{C}^{T} \tilde{V}_{2}^{-1} \hat{C}-B \tilde{R}_{2}^{-1}\left(\hat{B}^{T} P \hat{A}+R_{12}^{T}\right)-\hat{B} D_{c} \hat{C}\right] G^{T} \\
& B_{c}=\Gamma\left[\hat{A} Q \hat{C}^{T} \tilde{V}_{2}^{-1}+\hat{B} D_{c}\right] \\
& C_{c}=-\left[\tilde{R}_{2}^{-1}\left(\hat{B}^{T} P \hat{A}+\hat{R}_{12}^{T}\right)+D_{c} \hat{C}\right] G^{T} \\
& D_{c}=-\hat{R}_{2}^{-1}\left(\hat{B}^{T} P \hat{A} Q \hat{C}^{T}+\hat{R}_{12}^{T} Q \hat{C}^{T}\right) \tilde{V}_{2}^{-1}
\end{aligned}
$$

and such that $Q, P, \hat{Q}$, and $\hat{P}$ satisfy

$$
\begin{aligned}
& Q=\hat{A} Q \hat{A}^{T}-\hat{A} Q \hat{C}^{\tau} \hat{V}_{2}^{-1} \hat{C} Q \hat{A}^{T}+\hat{V}+\tau_{\perp} \hat{Q} \tau_{1}^{T}, \\
& P=\hat{A}^{T} P \hat{A}-\left(\hat{B}^{T} P \hat{A}+\hat{R}_{12}^{T}\right)^{T} \hat{R}_{2}^{-1}\left(\hat{B}^{T} P \hat{A}+\hat{R}_{12}^{T}\right)+\hat{R}_{1}+\tau_{\perp}^{T} \hat{P}_{\perp}, \\
& \hat{Q}=\hat{A}_{P} \tau \hat{Q} \tau^{T} \hat{A}_{P}^{T}+\Sigma_{Q}, \\
& \hat{P}=\hat{A}_{Q}^{T} \tau^{T} \hat{P}_{\tau} \hat{A}_{Q}+\Sigma_{P},
\end{aligned}
$$

where

$$
\tau=\sum_{i=1}^{n_{c}} \Psi E_{i} \Psi^{-1}
$$

for some $\Psi \in \mathcal{C}(\hat{Q}, \hat{P})$ such that $\left(\Psi^{-1} \hat{Q} \hat{P} \Psi\right)_{(i, i)} \neq 0, i=1, \cdots, n_{c}$, and some projective factorization $G, \Gamma$ of $\tau$. Furthermore, the minimal cost is given by

$$
J\left(A_{c}, B_{c}, C_{c}, D_{c}\right)=\delta+\operatorname{tr}\left[\left(M Q M^{T}+\hat{M} \tau \hat{Q} \tau^{T} \hat{M}^{T}\right) \hat{R}\right] .
$$

Remark 3.1: Theorem 3.1 can immediately be specialized to the more restrictive problem in which the compensator is strictly proper. This can be done in both the full- and reduced-order cases by ignoring (3.8) and setting $D_{c}=0$ wherever it appears. See [15], [16].

\section{NUMERICAL EVALUATION OF INTEGRALS INVOLYING MATRIX EXPONENTLALS}

To evaluate the exponential/integral expressions appearing in Theorem 2.1, we utilize the approach of [20]. The idea is to eliminate the need for integration by computing the matrix exponential of appropriate block matrices. Numerical matrix exponentiation is discussed in [21].

Proposition 4.1: Consider the following partitioned matrix exponentials of order $(3 n+l) \times(3 n+l),(3 n+m) \times(3 n+m),(2 n+m)$ $\times(2 n+m)$, and $(3 n) \times(3 n)$, respectively:

$$
\begin{aligned}
& {\left[\begin{array}{cccc}
F_{1} & F_{2} & F_{3} & F_{4} \\
0_{n} & F_{5} & F_{6} & F_{7} \\
0_{n} & 0_{n} & F_{8} & F_{9} \\
0_{l \times n} & 0_{l \times n} & 0_{l \times n} & I_{I}
\end{array}\right] \triangleq \exp \left[\begin{array}{cccc}
-A & I_{n} & 0_{n} & 0_{n \times l} \\
0_{n} & -A & V_{1} & V_{12} \\
0_{n} & 0_{n} & A^{T} & C^{T} \\
0_{l \times n} & 0_{l \times n} & 0_{l \times n} & 0_{l}
\end{array}\right] h,} \\
& {\left[\begin{array}{cccc}
F_{10} & F_{11} & F_{12} & F_{13} \\
0_{n} & F_{14} & F_{15} & F_{16} \\
0_{n} & 0_{n} & F_{17} & F_{18} \\
0_{m \times n} & 0_{m \times n} & 0_{m \times n} & I_{m}
\end{array}\right] \triangleq \exp \left[\begin{array}{cccc}
-A^{T} & I_{n} & 0_{n} & 0_{n \times m} \\
0_{n} & -A^{T} & R_{1} & R_{12} \\
0_{n} & 0_{n} & A & B \\
0_{m \times n} & 0_{m \times n} & 0_{m \times n} & 0_{m}
\end{array}\right] h,} \\
& {\left[\begin{array}{ccc}
I_{n} & F_{19} & F_{20} \\
0_{n} & F_{21} & F_{22} \\
0_{m \times n} & 0_{m \times n} & I_{m}
\end{array}\right] \triangleq \exp \left[\begin{array}{ccc}
0_{n} & I_{n} & 0_{n \times m} \\
0_{n} & A & B \\
0_{m \times n} & 0_{m \times n} & 0_{m}
\end{array}\right] h \text {, }} \\
& {\left[\begin{array}{ccc}
F_{23} & F_{24} & F_{25} \\
0_{n} & F_{26} & F_{27} \\
0_{n} & 0_{n} & F_{28}
\end{array}\right] \triangleq \exp \left[\begin{array}{ccc}
-A & I_{n} & 0_{n} \\
0_{n} & -A & V_{1} \\
0_{n} & 0_{n} & A^{T}
\end{array}\right] h \text {. }}
\end{aligned}
$$

Then

$$
\begin{gathered}
A^{\prime}=F_{17}, B^{\prime}=F_{18}, C^{\prime}=\frac{1}{h} F_{9}^{T}, D^{\prime}=\frac{1}{h} C F_{20}+D, \delta=\frac{1}{h} \text { tr } R_{1} F_{28}^{T} F_{25}, \\
V_{1}^{\prime}=F_{8}^{T} F_{6,}, V_{12}^{\prime}=\frac{1}{h} F_{8}^{T} F_{7}, \quad V_{2}^{\prime}=\frac{1}{h}\left(V_{2}+\frac{1}{h} C F_{8}^{T} F_{4}+\frac{1}{h} F_{4}^{T} F_{8} C^{T}\right), \\
R_{i}^{\prime}=\frac{1}{h} F_{17}^{T} F_{15}, R_{12}^{\prime}=\frac{1}{h} F_{17}^{T} F_{16}, R_{2}^{\prime}=R_{2}+\frac{1}{h}\left(B^{T} F_{17}^{T} F_{13}+F_{13}^{T} F_{17} B\right)
\end{gathered}
$$

\section{NuMERICAL SOLUTION OF THE DISCRETE-TIME OPTIMAL PROJECTION EQUATIONS}

The following algorithm is proposed for solving (3.9)-(3.12).

\section{Algorithm 5.1:}

Step 1: Initialize $k=0$ and $\tau^{(0)}=I_{n+1}$.

Step 2: With $\tau \triangleq \tau^{(k)}$ solve (3.9)-(3.12) for $Q^{(k)} \triangleq Q, P^{k)} \triangleq P, \hat{Q}^{(k)}$ $\triangleq \hat{Q}$, and $\hat{P}^{(k)} \triangleq \hat{P}$.

Step 3: If $k \geq 1$ check for convergence: If $\|\left(Q^{(k)}, P^{(k)}, \hat{Q}^{(k)}, \hat{P}^{(k)}\right)-$ $\left(Q^{(k-1)}, P^{(k-1)}, \hat{Q}^{(k-1)}, \hat{P}^{(k-1)}\right) \|>$ tol then continue; else go to Step 6 .

Step 4: Select $\Psi^{(k)} \in \mathcal{C}\left(\hat{Q}^{(k)}, \hat{P}^{(k)}\right)$ and update $\tau^{(k+1)}=\sum^{n_{c}}{ }_{i=1}$ $\Psi^{(k)} E_{j}\left(\Psi^{(k)}\right)^{-1}$.

Step 5: Increment $k$ and go to Step 2.

Step 6: Evaluate (3.5)-(3.8) with $Q=Q^{(k)}, P=P^{(k)}, \hat{Q}=\hat{Q}^{(k)}, \hat{P}$ $=\hat{P}^{(k)}, G^{T} \Gamma=\tau^{(k)}, \Gamma G^{T}=I_{n_{c^{*}}}$.

Remark 5.1: In solving the Riccati equation (3.9), the nonhomogeneous term is taken to be $\hat{V}+\tau_{\perp} \hat{Q}_{T}^{T}$, which is nonnegative definite. Similar remarks apply to (3.10). 
TABLE I

COST $J$ FOR BEAM EXAMPLE

\begin{tabular}{|c|c|c|c|}
\hline & 0 & .1 & .5 \\
12 & & & \\
10 & 1.1677 & 1.3723 & 3.0134 \\
8 & 1.1883 & 1.3823 & 3.0162 \\
6 & 1.2086 & 1.4421 & 3.0195 \\
4 & 1.3330 & 1.5752 & 3.0812 \\
2 & 1.4789 & 2.0425 & 3.3406 \\
& Open-Loop Cost (u=0) is 101.73 \\
\hline
\end{tabular}

Remark 5.2: The critical step of Algorithm 5.1 is the choice of $\Psi^{(k)}$ for constructing the projection $\tau^{(k+1)}$. Since $\Psi^{(k)}$ can include basis rearrangements, the choice of $\Psi^{(k)}$ essentially corresponds to a selection of $n_{c}$ rank1 eigenprojections out of $n+l$ possible eigenprojections. This selection is discussed at length in [22] where it is pointed out that the choice of eigenprojections determines which local extremal will be reached by the algorithm. Component-cost methods have thus been utilized as a promising selection criteria. Because of the eigenprojection structure of the necessary conditions, Algorithm 5.1 is fundamentally different from gradient search methods.

\section{Illustrative EXAMPLE: ConTROL OF A FleXiBle BEAM}

Consider a simply supported beam of length two with two colocated sensor/actuator pairs placed at coordinates $a_{1}=55 / 172$ and $a_{2}=45 / 43$. Define

$$
\begin{aligned}
& A=\underset{i=1, \cdots, s}{\operatorname{block}-\operatorname{diag}}\left[\begin{array}{cc}
0 & 1 \\
-\omega_{i}^{2} & -2 \zeta \omega_{i}
\end{array}\right], \omega_{i}=i^{2}, i=1, \cdots, 5, \zeta=0.005 \\
& B_{(i j)}=0.5(-1)^{j+1}\left(1+(-1)^{i}\right) \sin \left(i \pi a_{j} / 4\right), \\
& i=1, \cdots, 10, j=1,2, C=B^{T}, \\
& V_{1}=\underset{i=1, \cdots, 5}{\operatorname{block}-\operatorname{diag}}\left[\begin{array}{ll}
0 & 0 \\
0 & 1
\end{array}\right], V_{12}=0, V_{2}=0.1 I_{2} \\
& R_{1}=\underset{i=1, \cdots, 5}{\text { block-diag }}\left[\begin{array}{cc}
1 & 0 \\
0 & \omega_{i}^{-2}
\end{array}\right], R_{12}=0, R_{2}=0.1 I_{2} \text {. }
\end{aligned}
$$

For $n_{c}=10,8,6,4,2$ continuous-time controllers were designed using the results of [17] and, for $n_{c}=12,10,8,6,4,2$ and $h=0.1,0.5$, strictly proper $\left(D_{c}=0\right)$ discrete-time controllers were obtained from Theorem 3.1. The results are summarized in Table I.

\section{DIRECTIONS FOR FURTHER DEVELOPMENTS}

The following extensions and related developments immediately suggest themselves: reduced-order, discrete-time modeling/estimation of continuous-time systems [22], [23]: robust sampled-data control of uncertain systems with multiplicative noise [24]-[27]; multirate sampling [28], [29]; alternative $\mathrm{A} / \mathrm{D}$ and $\mathrm{D} / \mathrm{A}$ devices and asynchronous sampling/ control update; infinite-dimensional systems [30], [31].

\section{REFERENCES}

[1] J. R. Ragazzini and G. F. Franklin, Sampled-Data Control Systems. New York: McGraw-Hill, 1958.

[2] E. I. Jury, Sampled-Data Control Systems. New York: Wiley: 1958.

[3] J. T. Tou, Digital and Sampled-Data Control Systems. New York: McGrawHill, 1959.

[4] A. J. Munroe, Digital Processes for Sampled Data Systems. New York: Wiley, 1962.

[5] B. C. Kuo, Analysis and Synthesis of Sampled-Data Control Systems. Englewood Cliffs, NJ: Prentice-Hall, 1963.

[6] D. P. Lindorff, Theory of Sampled-Data Control Systems. New York: Wiley, 1964.

[7] G. F. Franklin and J. D. Powell, Digital Control of Dynamic Systems. Reading, MA: Addison-Wesley, 1980.

[8] K. J. Astrom, Introduction to Stochastic Control Theory. New York: Academic, 1970.

[9] A. H. Levis, M. Athans, and R. Schlueter, "On the behavior of optimal linear sampled-data regulators," Int. J. Contr., vol. 13, pp. 343-361, 1971.

[10] P. Dorato and A. Levis, "Optimal linear regulators: The discrete case," IEEE Trans. Auromat. Contr., vol. AC-16, pp, 613-620, 1971.

[11] K. Kwakemaak and R. Sivan, Linear Optimal Control Systems. New York: Wiley, 1972.

[12] N. Halyo and A. K. Caglayan, "A separation theorem for the stochastic sampleddata LQG problem," Int. J. Contr., vol. 23, pp. 237-244, 1976.

[13] E. S. Armstrong and A. K. Caglayan. "An algorithm for the weighting matrices in the sampled-data optimal linear regulator problem," NASA Langley, Hampton, VA, Rep. NASA TN D-8372, 1976.

[14] T. Mita, "Optimal digital feedback control systems counting computation time of control laws," IEEE Trans. Automat. Contr., vol. AC-30, pp. 542-548, I985.

[15] D. S. Bernstein, L. D. Davis, S. W. Greeley, and D. C. Hyland, "The optimal projection equations for reduced-order, discrete-time modelling, estimation and control," Proc. 24th IEEE Conf. Dec. Contr., Fort Lauderdale, FL. Dec. 1985, pp. 573-578.

[16] D. S. Bernstein, L. D. Davis, and D. C. Hyland, "The optimal projection equations for reduced-order, discrete-time modelling, estimation and control," $J$. Guid. Conir. Dynam., vol. 9, pp. 288-293, 1986.

[17] D. C. Hyland and D. S. Bernstein, "The optimal projection equations for fixedorder dynamic compensation," IEEE Trans. Automat. Contr., vol. AC-29, pp. 1034-1037, 1984.

[18] T. Kato, Perturbation Theory for Linear Operators. 2nd ed. New York: Springer-Verlag, 1976.

[19] C. R. Rao and S. K. Mitra, Generalized Inverse of Matrices and Its Applications. New York: Wiley, 1971.

[20] C. F. Van Loan, "Computing integrals involving the matrix exponential," IEEE Trans. Aufomat. Contr., vol. AC-23, pp. 395-404, 1978.

[21] C. Moler and C. Van Loan, "Nineteen dubious ways to compute the exponential of a matrix," SIAM Rev., vol. 20, pp. 801-836, 1978.

[22] D. C. Hyland and D. S. Bernstein, "The optimal projection equations for model reduction and the relationships among the methods of Wilson, Skelton, and Moore," IEEE Trans. Automat. Contr., vol. AC-30, pp. 1201-1211, 1985.

[23] _- "The optimal projection equations for reduced-order state estimation," IEEE Trans. Automat. Contr., vol. AC-30, pp. 583-585, 1985.

[24] D. S. Bernstein and D. C. Hyland, "Optimal projection/maximum entropy stochastic modelling and reduced-order design synthesis," in Proc. IFAC Workshop on Model Error Concepts and Compensation, Boston, MA, June 1985 .

[25] — " "The optimal projection/maximum entropy approach to designing loworder, robust controllers for flexible structures" in Proc. 24th IEEE Conf. Decision Contr., Fort Lauderdale, FL, Dec. 1985, pp. 745-752.

[26] _-, "The optimal projection equations for reduced-order model]ing, estimation and control of linear systems with Stratonovich multiplicative white noise," submitted for publication.

[27] A. R. Tiedemann and W. L. DeKoning, "The equivalent discrete-time optimal control problem for continuous-time systems with stochastic parameters, "Int. J Contr., vol. 70, pp. 449-466, 1984.

[28] D. P. Glasson, "A new technique for multirate digital control design and sample rate selection," J. Guid. Contr. Dynam., vol. 5, pp. 379-382, 1982.

[29] M. Araki and K. Yamamoto, "Multivariable multirate sampled-data systems: State-space description, transfer characteristics, and Nyquist criterion," IEEE Trans, Automat. Contr., vol. AC-31, pp. 145-154, 1986.

[30] M. J. Balas, "The structure of discrete-time finite-dimensional control of distributed parameter systems," J. Math. Anal. Appl., to be published.

[31] D. S. Bernstein and D. C. Hyland, "The optimal projection equations for finitedimensional fixed-order dynamic compensation of infinite-dimensional systems," SIAM J. Contr. Optimiz., vol. 24, pp. 122-151, 1986. 